\title{
Intramolecular Electron Transfer in Biferrocene Monocation: Evaluation of Franck-Condon Effects via a Time-Dependent Analysis of Resonance Raman Scattering in the Extended Near-Infrared
}

\author{
Robert D. Williams, Vladimir I. Petrov, Hong Peter Lu, and Joseph T. Hupp* \\ Department of Chemistry, Northwestern University, Evanston, Illinois 60208
}

Received: October 25, 1996; In Final Form: August 12, $1997^{\otimes}$

\begin{abstract}
Franck-Condon effects associated with intramolecular electron transfer in the biferrocene monocation have been evaluated via time-dependent analyses of resonance Raman scattering intensities and the intervalence absorption line shape. Quantitative studies in the extended near-infrared (1320 and $1064 \mathrm{~nm}$ excitation) reveal that three vibrational modes are displaced in the electron-transfer process. The most significant of these, the symmetrical ring- $\mathrm{Fe}$-ring stretch, has also been implicated in prior X-ray crystallographic studies. Mode or bond displacements obtained from the Raman analysis are slightly larger than estimates based on crystallographic displacements. These differences together with contributions from two additional modes identified by Raman appear to account for seemingly disparate estimates of vibrational reorganization energies provided by (a) the crystallographic method and (b) "Pekar plot" intercepts derived from intervalence absorption maxima. Implications for electron exchange kinetics are also discussed.
\end{abstract}

\section{Introduction}

The ferrocenium/ferrocene redox couple is an archetypal example of a class of metallocene couples that has proven exceptionally useful for understanding electron-transfer kinetics and dynamics in solution, ${ }^{1}$ at electrochemical interfaces,${ }^{2}$ and in crystalline solid-state environments. ${ }^{3}$ Studies with these systems have provided important experimental insight into the roles of solvent dynamics, ${ }^{1}$ lattice dynamics, ${ }^{3}$ barrier effects, ${ }^{4}$ ion-pairing effects, ${ }^{5}$ encapsulation effects, ${ }^{6}$ electronic coupling effects, ${ }^{4,7,8}$ and long-range electron-tunneling effects. ${ }^{2}$ One of the tools for interpreting these effects has been the assessment of intervalence absorption spectra for the dimeric mixed-valence form of the parent ferrocenium/ferrocene couple.,5a,b,6a,7 For example, the intervalence absorption maximum, $E_{\mathrm{op}}$, provides an approximate measure of the total reorganization energy, $\chi$, for intramolecular electron transfer (eq 1), while its solvent dependence permits a rough partitioning of the energy into solvational and vibrational parts. ${ }^{9}$

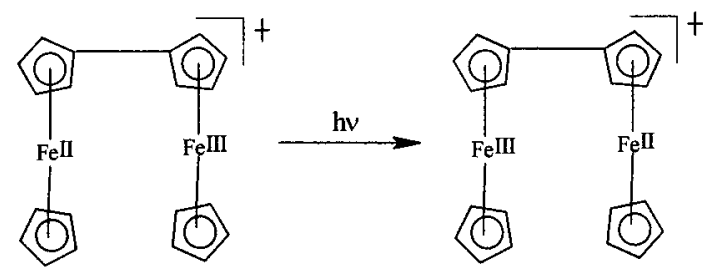

Here we report on a resonance Raman study of the intervalence charge-transfer process. Subsequent implementation of a spectral analysis based on wave packet propagation techniques permits absolute normal coordinate displacements and modespecific contributions to the vibrational reorganization energy to be determined. ${ }^{10}$ Information of this kind is important in evaluating the possible kinetic significance of nonclassical (highfrequency) vibrational effects. The study additionally provides some insight into apparent discrepancies between vibrational barriers determined via crystallographic measurements ${ }^{3}$ and barriers derived from absorption measurements. ${ }^{\text {ta }}$ Because the

${ }^{\otimes}$ Abstract published in Advance ACS Abstracts, October 1, 1997. intervalence transitions for symmetrical systems occur at very low energies, the Raman study has been implemented in the extended near-infrared (1320 nm excitation). Intervalence enhanced Raman scattering has been profitably employed previously with unsymmetrical systems (visible light absorbers) ${ }^{11}$ and with class III (valence-delocalized) systems. ${ }^{12}$ However, a previous attempt to examine symmetrical class II (valence-localized) systems by intervalence enhanced Raman scattering with $1064 \mathrm{~nm}$ excitation was thwarted by strong preresonance scattering from higher energy transitions. ${ }^{13}$ To the best of our knowledge, this report is the first to describe a successful application of the strategy to a symmetrical valencelocalized system.

\section{Experimental Section}

Resonance and normal Raman scattering measurements were made on a Spex 1403 double monochromator containing gratings blazed at $1200 \mathrm{~nm}(600$ groove/mm $)$. The mechanically chopped output of an Nd:YAG laser (Lee Laser 712ST) operating at either $1064 \mathrm{~nm}$ or 1320 and $1337 \mathrm{~nm}$ was used for sample excitation. Typical powers were 1.1 and $0.8 \mathrm{~W}$ at the shorter and (combined) longer wavelengths, respectively. Samples were irradiated in a spinning NMR tube $\left(90^{\circ}\right.$ geometry) while cooling with a stream of nitrogen. Detection was achieved with a liquid nitrogen cooled Ge detector $(4 \times 4 \mathrm{~mm}$; North Coast Model EO817L) coupled to a Stanford Instruments Model SR530 lock-in amplifier. To eliminate spectral artifacts from water vapor absorption, ${ }^{12 \mathrm{~b}}$ the entire monochromator was purged with dry nitrogen. For 1320/1337 nm experiments, no attempt was made to separate the dual excitation colors. Consequently, scattering peaks were duplicated with $110 \mathrm{~cm}^{-1}$ separation.

Prior to analysis, Raman spectra were corrected for instrument throughput and detector response by calibrating the overall response with an approximate blackbody source $(100 \mathrm{~W}$ quartz halogen lamp (Epply Laboratory, Inc.); Power Ten Model 3300B-4015 power supply designed for spectral calibration). Self-absorption corrections were made by comparison of relative solvent intensities in pure solvent and biferrocene-containing solutions. Electronic absorption spectra were obtained on an OLIS-modified Cary-14 spectrophotometer. 


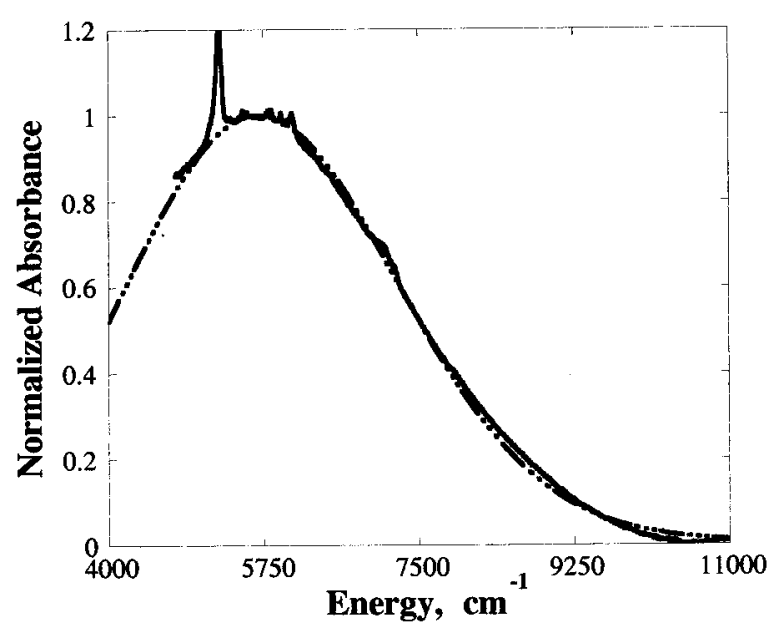

Figure 1. Experimental (solid line) and simulated (dashed line) intervalence absorption spectra for biferrocene monocation in $\mathrm{CD}_{3} \mathrm{CN}$.

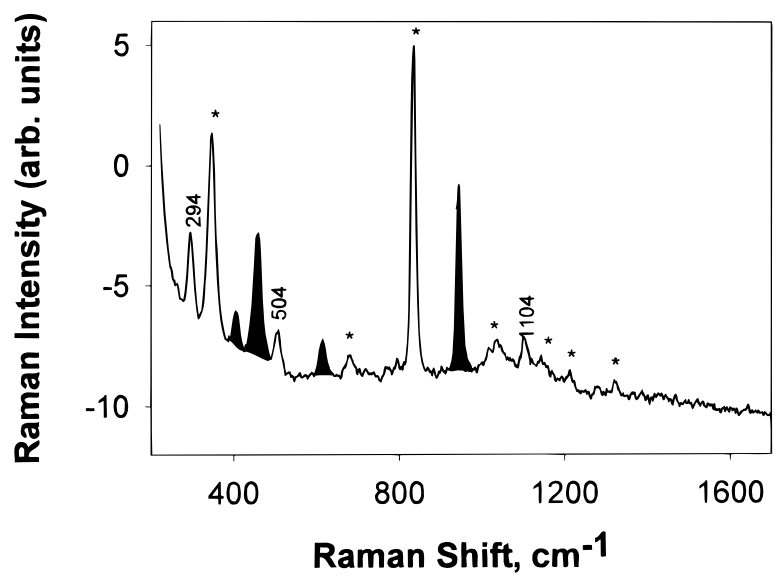

Figure 2. Resonance Raman spectrum for biferrocene monocation in $\mathrm{CD}_{3} \mathrm{CN}$, based on dual excitation at 1320 and $1337 \mathrm{~nm}$. Peaks associated with the latter are shaded. Asterisks denote solvent peaks. All Raman shifts are reported relative to the $1320 \mathrm{~nm}$ excitation line.

Acetonitrile- $d_{3}$ was obtained from Aldrich and used as received. Acetonitrile- $h_{3}$ was obtained from Fisher and distilled over $\mathrm{CaH}_{2}$ before use. Biferrocene was kindly provided by Prof. Roger Nielson of Southern Oregon State College.

Samples of the mixed-valence ion were prepared in acetonitrile by oxidation of the fully reduced form with either bromine vapor or $\left[\mathrm{Fe}\left(2,2^{\prime} \text {-bipyridine }\right)_{3}\right]\left(\mathrm{PF}_{6}\right)_{3}$. Concentrations varied from $<1$ to $\sim 10 \mathrm{mM}$, with the best Raman spectra being obtained at higher concentrations. Intervalence absorption spectra, on the other hand, were obtained at lower concentrations in order to minimize inhomogeneous broadening effects associated with ion pairing. The majority of the absorption and scattering spectra were obtained in acetonitrile- $d_{3}$ in order to avoid deleterious $\mathrm{C}-\mathrm{H}$ overtone absorption at the furthest red wavelengths.

\section{Results}

Spectra. Shown in Figure 1 is the portion of the electronic absorption spectrum associated with the intervalence transition (eq 1). The observed bandwidth and band maximum agree reasonably well with earlier reports. ${ }^{4}$ Figure 2 displays the Raman scattering spectrum for the biferrocene cation based on $1320 / 1337 \mathrm{~nm}$ excitation. We observed three modes assignable to the mixed valence ion. The intense mode at $294 \mathrm{~cm}^{-1}$ is assigned to the symmetrical cyclopentadiene-Fe-cyclopentadiene (cp) stretch $\left(v_{4}\right.$ in ferrocene). ${ }^{14}$ In principle, this mode (as well as the others) should appear twice in the scattering
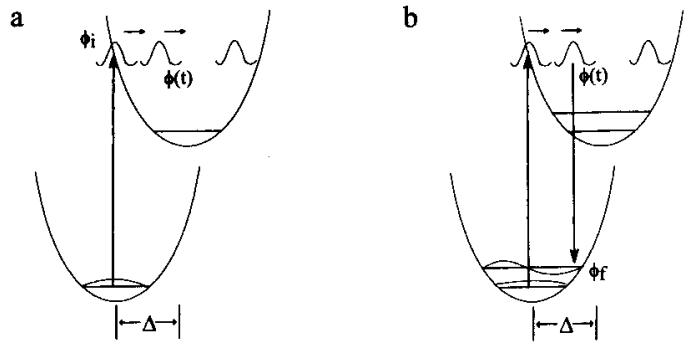

Figure 3. Schematic representation of wave packet propagation descriptions of (a) absorption and (b) resonance Raman scattering processes.

spectrum because of oxidation-state-induced frequency shifts and the necessary involvement of both $\mathrm{Fe}$ (II) and $\mathrm{Fe}$ (III) centers in the intramolecular electron-transfer process. Given the comparatively low resolution (ca. $7 \mathrm{~cm}^{-1}$ ) of spectra generated in our experiments, observation of only a single stretching peak is consistent with the reported near coincidence of $\mathrm{cp}-\mathrm{Fe}-\mathrm{cp}$ stretching frequencies for isolated ferrocene versus ferrocenium. ${ }^{15}$ The $504 \mathrm{~cm}^{-1}$ mode is tentatively assigned to a $\mathrm{cp}-$ $\mathrm{Fe}-\mathrm{cp}$ bending motion $\left(v_{21}\right.$, found at $492 \mathrm{~cm}^{-1}$ in ferrocene and $510 \mathrm{~cm}^{-1}$ in ferrocenium $\left.{ }^{16}\right)$, a vibration which, according to recent normal coordinate analyses, ${ }^{17}$ also has some ring metal-ring bending character ( $v_{22}$ in ferrocene). Further evidence for this motion can be found in one of the crystal structures (in which the $\mathrm{cp}-\mathrm{Fe}^{\mathrm{II}}-\mathrm{cp}$ angle is $\sim 180^{\circ}$, but the $\mathrm{cp}-\mathrm{Fe}^{\mathrm{III}}-\mathrm{cp}$ angle is only $\left.\sim 175^{\circ}\right){ }^{36 \mathrm{a}}$ Finally, the $1100 \mathrm{~cm}^{-1}$ mode is assigned to a ring breathing motion ( $v_{3}$ in ferrocene). ${ }^{18}$ That all three modes are resonantly enhanced by the intervalence transition was established by comparison to experiments performed off-resonance (1064 $\mathrm{nm}$ excitation) and by comparison to experiments involving the neutral form of biferrocene (transparent at $1320 \mathrm{~nm}$; no scattering observed). After correction for instrument throughput, detector response, and sample self-absorption effects, the 294 and $1100 \mathrm{~cm}^{-1}$ modes were found to have $93 \%$ and $13 \%$, respectively, of the intensity of the $504 \mathrm{~cm}^{-1}$ mode.

Time-Dependent Analysis. In principle, both absorption line shapes and resonance Raman scattering intensities contain information about ground-state/excited-state normal coordinate displacements, $\Delta$, and therefore, vibrational reorganization energies. Computationally tractable, quantitative relationships among these parameters can be formulated by using standard time-dependent treatments of electronic and vibrational spectroscopy. ${ }^{10}$ Particularly useful from our perspective is Heller's formulation $^{10 \mathrm{c}}$ of the time-dependent theory, which treats both the absorption and scattering processes as frozen Gaussian wave packet propagation problems (Figure 3a). Briefly, both processes involve projection of an initially stationary vibrational wave packet on the ground electronic state surface up to an excited surface, where the packet evolves in time. As shown in eq 2 , the absorbance spectrum in the time domain can then

$$
\begin{aligned}
&\left\langle\phi_{\mathrm{i}} / \phi(t)\right\rangle=\exp \left\{\sum _ { \kappa } \left[\frac{1}{2} \Delta_{k}^{2}\left(1-\exp \left(-\mathrm{i} \omega_{k} t\right)\right]-\right.\right. \\
&\left.\mathrm{i} E_{00} t-\Gamma^{2} t^{2}-\chi_{\mathrm{s}} k T t^{2}-\mathrm{i} \chi_{\mathrm{s}} t\right\}
\end{aligned}
$$

be represented in terms of the overlap of the moving wave packet, $\phi(t)$, with a wave function, $\phi_{\mathrm{i}}$, describing the initial vibrational state. In the equation, $E_{00}$ is the electronic energy gap, the $\omega_{k}$ 's are mode frequencies, the $\Delta_{k}$ 's are unitless normal coordinate displacements, and $\Gamma$ is a damping or dephasing term that acts to kill wave packet recurrences on the upper electronic 
surface. Following Myers, ${ }^{10 a}$ two terms involving the solvent reorganization energy, $\chi_{\mathrm{s}}$, and the temperature, $T$, are also included. The first term (real term) acts as an inhomogeneous broadening term (similar to $\Gamma$ ), while the second term (imaginary term) accounts for the classical Franck-Condon activity of the solvent and, therefore, yields a spectral energy shift. A Fourier transform of the time domain spectrum yields the frequency domain spectrum:

$$
I(\omega)=C \omega \int_{-\infty}^{\infty} \mathrm{e}^{\mathrm{i} \omega t}\langle\phi / \phi(t)\rangle \mathrm{d} t
$$

For Raman scattering one is interested in the overlap of the time-evolving wave packet (now describing the initial state; Figure $3 b$ ) with a wave function, $\phi_{\mathrm{f}}$, describing a vibrationally excited final state on the ground electronic surface. For this process, the time-dependent behavior is expressed as

$$
\begin{aligned}
\left\langle\phi(t) / \phi_{\mathrm{f}}\right\rangle=\prod_{\kappa}\left\{\operatorname { e x p } \left[-\frac{\Delta_{k}^{2}}{2}\left(1-\exp \left(-\mathrm{i} \omega_{k} t\right)\right](1-\right.\right. \\
\left.\left.\exp \left(-\mathrm{i} \omega_{k} t\right)\right) n_{k} \times \frac{(-1)^{n} k \Delta_{k}^{n_{k}}}{\left(2^{n} k n_{k} !\right) 1 / 2}\right\} \exp \left(-\mathrm{i} E_{00} t\right)
\end{aligned}
$$

where $n_{k}$ is the final vibrational state (ground electronic surface) of the mode of interest. As shown in eq 5, the half-Fourier

$$
(\alpha)_{\mathrm{fi}}=\frac{\mathrm{i}}{\hbar} \int_{0}^{\infty}\langle\phi / \phi(t)\rangle \exp \left\{\mathrm{i} \omega t-\Gamma t-\mathrm{i} \chi_{\mathrm{s}} t-\chi_{\mathrm{s}} k T t^{2}\right\} \mathrm{d} t
$$

transform of this overlap gives the polarizability change $\left(\alpha_{\mathrm{fi}}\right)$ for each mode. The polarizability change, in turn, is related to the scattering intensity for that mode by

$$
I_{\mathrm{i} \rightarrow \mathrm{f}} \propto \omega_{\mathrm{I}} \omega_{\mathrm{s}}^{3}\left(\alpha_{\mathrm{fi}}^{*}\right)\left(\alpha_{\mathrm{fi}}\right)
$$

where $\omega_{\mathrm{I}}$ is the frequency of incident light, $\omega_{\mathrm{s}}$ is the absolute frequency of scattered light, and $\alpha_{\mathrm{fi}}{ }^{*}$ is a complex conjugate.

Despite the inclusion of temperature-dependent solvent terms, ${ }^{10 a}$ it should be noted that eqs $2-5$ correspond vibrationally to a "zero degree Kelvin" theory. Champion and coworkers have pointed out that neglect of finite-temperature effects in time correlator theories can lead to significant errors in calculated coordinate displacements for low-frequency vibrational modes. ${ }^{19}$ The errors arise because vibrational overlaps (and polarizability changes) scale as $(n+1)^{1 / 2}$, where $n$ is the initial vibrational level. Consequently, scattering arising from higher vibrational states is more intense than scattering arising from the ground state. More complex versions of the time correlator theory exist which take into account scattering from higher vibrational levels, ${ }^{20}$ but since only one mode is vibrationally hot in our experiments (i.e., the $\mathrm{cp}-\mathrm{Fe}-\mathrm{cp}$ stretch at $294 \mathrm{~cm}^{-1}$ ), we have taken a more approximate approach. The experimental Raman scattering intensity of the $294 \mathrm{~cm}^{-1}$ mode has been "corrected" to its low-temperature value, and the corrected value has been used in the fitting procedure described below. The magnitude of the intensity correction is $15 \%$ based on an $18 \%$ population of $n=1$ and a $4 \%$ population of $n=2$. Corresponding hot band corrections to the absorption line shape have been neglected but almost certainly are small.

Energy Parameters. Analysis implementation requires values for $\chi_{\mathrm{s}}$ and $E_{00}$. The former was estimated from published plots of the intervalence absorption energy maximum, $E_{\text {op }}$, versus the composite quantity, $1 / D_{\text {op }}-1 / D_{\mathrm{s}}$, where $D_{\text {op }}$ and $D_{\mathrm{s}}$ are the optical and static dielectric constants of the solvent. According to Marcus-Hush theory, ${ }^{9,21}$ the intercepts of these
TABLE 1

\begin{tabular}{lcccc}
\hline Frequency & $\begin{array}{c}\text { Relative } \\
\text { Scattering } \\
\text { Intensity } \\
\left(\mathrm{cm}^{-1}\right)\end{array}$ & $\begin{array}{c}\text { Calculated } \\
\text { Displacement }\end{array}$ & $\begin{array}{c}\chi_{\mathrm{k}}{ }^{\prime} \\
\left(\mathrm{cm}^{-1}\right)\end{array}$ & Assignment \\
\hline 294 & 1.00 & $3.4 \pm 0.5$ & $1700 \pm 500$ & $\mathrm{v}_{4}: \mathrm{cp}-\mathrm{Fe}-\mathrm{cp}$ stretch \\
504 & 1.08 & $2.0 \pm 0.3$ & $1000 \pm 300$ & \\
1100 & 0.14 & $0.30 \pm 0.05$ & $50 \pm 15$ &
\end{tabular}

plots ("Pekar plots") give, in the diabatic limit, the sum of the inner-sphere reorganization energy $\left(\chi_{\mathrm{i}}\right)$ and $E_{00}$, so that the remainder of the optical energy must come from the solvent $\left(\chi_{\mathrm{i}}\right.$ $\left.+\chi_{\mathrm{s}}+E_{00}=E_{\mathrm{op}}\right)$. For reaction 1 the estimated value for $\chi_{\mathrm{s}}$ in acetonitrile is $2200 \mathrm{~cm}^{-1}$. 22

Although the symmetry of the intervalence transition argues that $E_{00}$ should be nominally constrained to zero, spectra were simulated using $E_{00}$ values ranging from 0 to $500 \mathrm{~cm}^{-1}$. The $500 \mathrm{~cm}^{-1}$ upper limit arises from uncertainties in the precise electronic configuration of the optical intervalence excited state and from additional considerations outlined in the Discussion section.

Franck-Condon Parameters. To extract electron-transferinduced coordinate displacements from Raman spectra, we began by calculating excitation profiles via eq 6 . To motivate the calculations we made use of (a) independent estimates for $\chi_{\mathrm{s}}$ and $E_{00}$ and (b) arbitrary guesses for $\Delta_{k}$. Calculated scattering intensities based on $1320 \mathrm{~nm}$ excitation were then used to adjust the estimated displacements until the displacements yielded relative scattering intensities that matched the (relative) experimental intensities. ${ }^{23}$ The corresponding mode displacements were then linearly scaled until a good fit to the intervalence absorbance spectrum was obtained. After checking the consistency of these displacements against the experimental Raman intensities and, if necessary, further iteratively fitting the scattering and absorption, final values for the Franck-Condon parameters (normal coordinate displacements) were determined.

Figure 1 shows that a very good fit to the absorption line shape can be obtained. Table 1 lists the parameters used to obtain the fit. With the coordinate displacement parameters in hand, single-mode contributions to the vibrational reorganization energy were then calculated via eq 7 :

$$
\chi_{k}^{\prime}=0.5 \Delta_{k}^{2} v_{k}
$$

Table 1 lists the resulting parameters.

\section{Discussion}

Parameter Fitting. Before considering the displacement and reorganizational parameters themselves, some discussion of the underlying spectral fitting protocol is appropriate. In principle, the number of parameters available to accomplish the fits is large. Note, however, that several parameters ( $T$ and the $\omega_{k}$ 's) are independently fixed, and several others $\left(\chi_{\mathrm{s}}\right.$ and $\left.E_{00}\right)$ are constrained to a limited range of values.

As mentioned in the Results section, $E_{00}$ should-in the simplest case- be constrained to zero by the symmetry of the intervalence transition. However, ferrocenium has been shown to have ${ }^{2} E_{2 g}$ ground state which is split by spin-orbit coupling, 


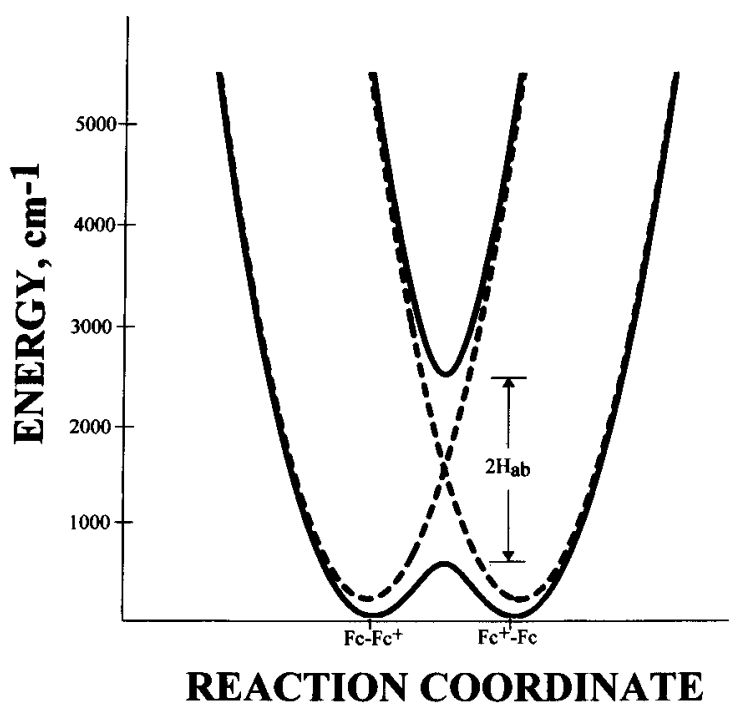

Figure 4. Diabatic and adiabatic potential energy surfaces for optical and thermal electron transfer in biferrocene monocation, assuming $\chi_{\text {total }^{-}}$ (adiabatic) $=4950 \mathrm{~cm}^{-1}$ and $H_{\mathrm{ab}}=900 \mathrm{~cm}^{-1}$.

a nonaxial ligand field, ${ }^{15,24}$ and a dynamic Jahn-Teller effect. ${ }^{24}$ From these splittings arise three possible intervalence excited states separated by about 500 wavenumbers in energy, ${ }^{25}$ resulting in an intervalence absorption which is very likely an overlap of at least three bands. ${ }^{26}$ The existence of multiple transitions presumably contributes at least several hundred wavenumbers of uncertainty to the experimentally relevant $E_{00}{ }^{27}$

An additional factor to be considered is that electronic coupling necessarily introduces an energy splitting between upper and lower adiabatic surfaces. The splitting, while numerically most significant in the diabatic curve crossing region (see Figure 4), is also finite in other regions. Interestingly, the splitting energy, while having a different physical significance, will play much the same role in eqs 2 and 5 as a conventional energy gap contribution (i.e. $E_{00}$ ). From first-order perturbation theory, the magnitude of the gap in the region pertinent to the intervalence absorption maximum (i.e. the lower surface minimum and the upper surface Franck-Condon region) is ${ }^{9 \mathrm{c}, 29}$

$$
E(\text { split })=\left(\chi^{2}+4 H_{\mathrm{ab}}^{2}\right)^{1 / 2}-\chi
$$

where $\chi$ is the total reorganization energy and $H_{\mathrm{ab}}$ is the nonadiabatic electronic coupling energy. To zeroth order, the total reorganization energy can be equated with $E_{\mathrm{op}}$. According to the generalized Mulliken-Hush theory, $H_{\mathrm{ab}}$ can be estimated experimentally from the ratio of the transition dipole moment to the change in dipole moment for the intervalence transition. ${ }^{28}$ Following Hush, ${ }^{9 a, 30}$ the latter is usually taken as the product of the unit electronic charge and the center-to-center donor/ acceptor separation distance. For intervalence excitation of biferrocene monocation, the estimated change in dipole moment would be $\sim 5.1$ e $\AA$, a value that is unrealistically high in view of the solvent reorganization energy. A more reasonable estimate is ca. $3.5 \mathrm{e} \AA{ }^{31}$ With this revised estimate, the transition dipole moment yields $900 \mathrm{~cm}^{-1}$ for $H_{\mathrm{ab}}$. From eq 7, $E$ (split) is then ca. $300 \mathrm{~cm}^{-1} \cdot 27$

In summary, the combination of strong electronic coupling and a nondegenerate ground state means that $E$ (split) $+E_{00}$ span approximately $500 \mathrm{~cm}^{-1}$ (with zero being the lower limit and $300 \mathrm{~cm}^{-1}$ representing the most reasonable value). In recognition of this, the Raman intensities and absorbance spectra were fit using a range of values for the composite energy quantity. An uncertainty of $500 \mathrm{~cm}^{-1}$ was also assumed for the $\chi_{\mathrm{s}}$. Fitting with the minimum values for both the composite energy and $\chi_{\mathrm{s}}$ results in the largest values for the normal coordinate displacements, while the smallest displacements result from fitting with the maximum values for both the composite energy and $\chi_{\mathrm{s}}$. The upper and lower limits of the reorganization energies (Table 1) result from these extreme fits.

The damping parameter, $\Gamma$, also was initially fixed at an arbitrarily small value $\left(10 \mathrm{~cm}^{-1}\right)$ in all of the fits, reflecting its presumed origin in dephasing effects. We discovered, however, that the intervalence line shape could be reproduced only after increasing $\Gamma$ to $500 \mathrm{~cm}^{-1}$ (even when fitting with the minimum values of the composite energy $\left(E_{00}+E\right.$ (split)) and $\left.\chi_{\mathrm{s}}\right)$. Large values for $\Gamma$ imply either (a) neglect of one or more modes suffering significant displacement or (b) unrecognized inhomogeneous broadening effects. The former was tested by addition of a fourth vibrational mode at $v=1600 \mathrm{~cm}^{-1}$. (This mode, if it existed, would fall beyond the red edge of the detector if the sample were excited at $1320 \mathrm{~nm}$.) Inclusion of the highfrequency mode, however, resulted in too broad an absorption spectrum, even when $\Gamma$ was reduced to $10 \mathrm{~cm}^{-1}$ (after rescaling the other displacements to account for the additional source of reorganization energy).

The alternate hypothesis-inhomogeneous broadening-also seems problematic, as ion pairing and solvent thermal effects (the most obvious sources of broadening) have already been avoided or taken into account. Another possibility is rotational isomerization about the ferrocene/ferrocenium single bond. Conceivably, this could introduce significant changes in the effective intervalence charge-transfer distance and, therefore, create additional uncertainty in both $\chi_{\mathrm{s}}$ and $E_{\mathrm{op}}$. A final possibility, of course, is that the various splittings giving rise to uncertainties in $E_{00}$ (see above) might also contribute to the composite bandwidth. This would, of course, require that multiple (overlapping) transitions contribute to the intervalence absorption intensity.

Another concern, of a more fundamental nature, is that electronic coupling in the biferrocene mixed-valence system might be great enough to invalidate the analysis described by eqs 2-6. Issues of this kind have been examined in some detail by Zink and co-workers. ${ }^{32}$ They point out that two related problems can exist. The first is that wave packet amplitude can be passed from the excited surface to the ground surface on each pass through the curve-crossing region of Figure 4. Phase differences between the resulting fragments now propagating simultaneously on the upper and lower surfaces can lead to interferences which are manifest both as dips in absorption spectra and as anomalous scattering intensities. Clearly, for interferences to occur the lifetime of the wave packet on the upper surface must be sufficient to permit at least one (and, preferably, multiple) vibrational recurrences to take place. ${ }^{33}$ This question was examined by recalculating the time domain spectrum without damping terms (to determine the vibrational period of the wave packet) and comparing that time with the time needed for complete decay (taken to be $90 \%$ of the maximum amplitude) of the wave packet overlap for scattering under full damping conditions. The time-dependent overlaps are illustrated in Figure 5. From the figure, the wave packet lifetime is sufficiently short that wave packet recurrences are effectively precluded and interference effects can be neglected. (The high-frequency component decays "completely" in $\sim 25 \%$ of a vibrational period, the medium-frequency component in $13 \%$, and the low-frequency component in $8 \%$.) We suggest that this might be a general result for charge-transfer transitions in solution because of the highly effective damping created by dissipative reorganization of the solvent. The best chance for 


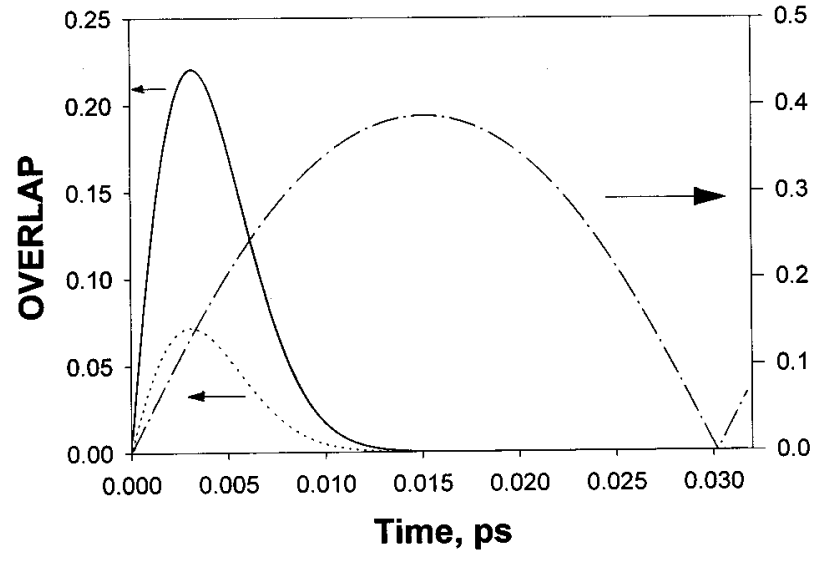

Figure 5. Overlaps for Raman excitation profiles (generated using eq 4) for the three different vibrations (294 and $504 \mathrm{~cm}^{-1}$ are nearly coincident, solid; $1100 \mathrm{~cm}^{-1}$, dotted) along with the overlap for 1100 $\mathrm{cm}^{-1}$ (dash-dot, scaled to right-hand axis) without influence from the other vibrations or damping terms.

observing these interesting effects, therefore, might come in studies of frozen solutions or in crystalline environments. ${ }^{3}$

The second problem is that the analysis assumes that wave packet motion occurs exclusively on the diabatic potential energy surfaces (dotted surfaces in Figure 4). The differences between diabatic and adiabatic surfaces are small in the FranckCondon region and at the bottom of the lower potential energy surface (Figure 4). The surfaces obviously diverge, however, in the vicinity of the avoided crossing. While the divergence is of little consequence for low-frequency modes (which move on shallow potential surfaces and sample only the FranckCondon region), it may be problematic for high-frequency wave packet motion, especially if the high-frequency component remains largely on the upper surface. To gauge this possibility, we implemented a simple Landau-Zener treatment ${ }^{34}$ of motion in the $1100 \mathrm{~cm}^{-1}$ coordinate. In this treatment, the critical parameters are the velocity of the high-frequency wave packet component, the steepness of the diabatic surfaces in the crossing region, and the magnitude of the gap to be crossed (i.e. $2 H_{\mathrm{ab}}$ ). ${ }^{35}$ From the treatment, the probability of crossing from the upper to the lower adiabatic surface (nonadiabatic crossing probability $P_{12}$ ) on a single pass is ca. $2 \%$ or less, and the wave packet is largely confined to the upper, adiabatic surface (In other words, this component of the wave packet moves on a surface that is not of the type assumed by eqs 2 and 4.). We conclude that the diabatic time-dependent analysis may indeed yield an incorrect value for the displacement of the $1100 \mathrm{~cm}^{-1}$ mode, but that it likely approximates well the displacements occurring in the much more highly Franck-Condon active (but lower frequency) modes at 294 and $504 \mathrm{~cm}^{-1}$.

Displacement Parameters and Crystallographic Comparisons. With the approximate validity of the time-dependent analysis established (at least for the low-frequency components of reaction 1), the structural details of the vibrational reorganization process can be usefully examined. Table 1 shows that the largest unitless coordinate displacement is associated with the symmetrical $\mathrm{cp}-\mathrm{Fe}-\mathrm{cp}$ stretch. From eq 8, the stretch contributes $1700 \pm 500 \mathrm{~cm}^{-1}$ to the reorganization energy. This displacement has also been implicated in several single-crystal X-ray structural studies. ${ }^{36}$ (Note, however, that the involvement of the two higher frequency modes has not.) Reported values of the $\mathrm{Fe}-\mathrm{C}$ bond displacement (10 bonds (or two cyclopentadienes) displaced per metallocene; two metallocenes per molecule) range from 0.04 to $0.065 \AA$ with reported uncertainties of $0.01-0.02 \AA .{ }^{36}$ If the bond length results are converted to normal coordinate displacements, the $\mathrm{X}$-ray experiments yield
1.9-3.1 for $\Delta(\mathrm{cp}-\mathrm{Fe}-\mathrm{cp}),{ }^{37}$ in rough agreement with the Raman experiment. From eq 8, this corresponds to a reorganizational energy contribution of $530-1400 \mathrm{~cm}^{-1}$.

Intervalence Absorption and Vibrational Reorganization Energies. According to Hush theory, ${ }^{9}$ the intervalence absorption energy maximum will equal the sum of vibrational, solvational, and electronic contributions:

$$
E_{\mathrm{op}}=\chi_{\mathrm{vib}}+\chi_{\mathrm{s}}+2 H_{\mathrm{ab}}^{2} / \chi_{\text {total }}
$$

If the relatively small electronic term $\left(\sim 300 \mathrm{~cm}^{-1}\right.$, cf. eq 7$)$ is neglected, the available solvent-dependent intervalence absorption measurements yield $\chi_{\text {vib }}$ values of $3500 \mathrm{~cm}^{-1}$. The substantial discrepancy between this value and X-ray-based estimates (ca. $1000 \pm 500 \mathrm{~cm}^{-1}$, above) has been noted. Much closer agreement is obtained between the sum of Ramandetermined vibrational contributions and the estimate from absorption measurements.

Electron-Transfer Kinetics. Finally, with the displacement and reorganizational parameters in hand, several calculations were done to assess the influence of Franck-Condon factors on electron-transfer rates. Here we considered the intermolecular ferrocene/ferrocenium electron exchange (equivalent to a unimolecular rate based on a fixed reactant separation). ${ }^{1}$ (Experimental data are unavailable for the intramolecular biferrocene self-exchange, at least in solution.) For the intermolecular reaction electronic coupling is probably sufficiently weak that barrier round off effects (adiabaticity effects; see Figure 4) can be ignored. In any case, a "classical" rate (corresponding to an activated barrier crossing) was calculated from $^{9 c}$

$$
k=\left(H_{\mathrm{ab}}{ }^{2} / \hbar\right)(\pi / \chi R T)^{1 / 2} \exp [-\chi / 4 R T]
$$

where $\chi$ is the total reorganization energy (determined by summing $\chi_{\mathrm{s}}$ and the $\chi_{k}^{\prime}$ values, where the $8040 \mathrm{~cm}^{-1}$ value of the former was taken from Weaver's work on ferrocene/ ferrocenium electron transfer, ${ }^{4 \mathrm{~d}}$ and the latter was taken from this work). Then, a "quantum" rate calculation (valid for an arbitrary number of modes) was made using 38,39

$$
\begin{gathered}
k=\frac{H_{\mathrm{ab}}^{2}}{\hbar}\left(\frac{\pi}{\chi_{\mathrm{s}} R T}\right)^{1 / 2} \exp \left(-\sum_{j} \mathrm{~s}_{j} \operatorname{coth}\left(\frac{\hbar \omega_{j}}{2 k T}\right)\right] \times \\
\prod_{j} \sum_{m_{j}=-\infty}^{+\infty} \exp \left(\frac{m_{j} \hbar \omega_{j}}{2 k T}\right) I_{m_{j}}\left\{S_{j} \operatorname{csch}\left(\frac{\hbar \omega_{j}}{2 k T}\right)\right\} \times \\
\exp \left[\frac{\left(\Delta E+\sum_{j} m_{j} \hbar \omega_{j}+\chi_{\mathrm{s}}\right)^{2}}{4 \chi_{\mathrm{s}} R T}\right]
\end{gathered}
$$

(Both equations describe reactivity within an electron-transfer precursor complex and therefore yield rate constants with units of $\mathrm{s}^{-1}$.) The ratio of quantum to classical rates was 1.3. Note that the rate ratio approach permits us to use arbitrary values for the nonadiabatic electronic coupling energy.

Rate calculations (eq 11) were also made without inclusion of the two additional vibrational modes uncovered by the Raman experiment. These calculations were done by using both the Raman-determined displacement for the $\mathrm{cp}-\mathrm{Fe}-\mathrm{cp}$ mode and the displacement inferred from crystallographic measurements. ${ }^{40}$ The rate constant was also calculated (eq 10) with vibrational contributions completely omitted. Table 2 shows that the 
TABLE 2

\begin{tabular}{llc}
\hline $\begin{array}{c}\text { type of } \\
\text { calculation }\end{array}$ & \multicolumn{1}{c}{ reorganization energy included } & $\begin{array}{c}\text { relative rate } \\
\text { attenuation } \\
\left(k_{\text {solvent }} / k\right)\end{array}$ \\
\hline classical $^{a}$ & solvent only & 1 \\
classical $^{a}$ & solvent +3 Raman modes & $c$ \\
quantum $^{b}$ & solvent +3 Raman modes $^{c}$ & 32 \\
quantum $^{b}$ & solvent $+294 \mathrm{~cm}^{-1}$ mode (Raman $)^{c}$ & 25 \\
quantum $^{b}$ & solvent $+294 \mathrm{~cm}^{-1}$ mode (crystallography) $)^{d}$ & 8 \\
\end{tabular}

${ }^{a}$ Calculated via eq $10 .{ }^{b}$ Calculated via eq $11 .{ }^{c}$ Displacement parameters taken from Table 1. ${ }^{d} \Delta(294)=2.76$ (see refs 36 and 37 and text).

various vibrational effects are relatively small, consistent with the idea $^{1}$ that the dominant reorganizational contribution, particularly for the intermolecular reaction, comes from the solvent.

\section{Conclusions}

Resonance-enhanced Raman scattering in the extended nearinfrared provides information about the vibrational modes coupled to intervalence charge transfer. A time-dependent analysis of the scattering spectrum and the corresponding absorption spectrum gives semiquantitative information about mode displacements and single-mode contributions to the reorganizational energy. The Raman experiments show that three vibrational modes contribute, with the largest contribution originating from a symmetrical ring-metal-ring stretching motion. From radiationless decay theory the modes appear to attenuate the ferrocene/ferrocenium self-exchange rate by about a factor of 30 in comparison to a hypothetical rate for a reaction featuring only a solvent barrier. ${ }^{41}$ The displacement analysis and rate calculations additionally show that only a modest nonclassical rate effect is likely to exist. Finally, the Raman analysis provides a partial explanation for the significant discrepancy between vibrational reorganization energies inferred from crystallographic measurements versus those generated from solvent-dependent absorption measurements. ${ }^{42}$

Acknowledgment. We thank Prof. Ken Spears for providing the quantum mechanical rate program, Prof. Mark Ratner for helpful discussions regarding wave packets, and a reviewer for insightful comments on spectral assignments. We thank the U.S. Department of Energy, Office of Energy Research, Division of Chemical Sciences (Grant No. DE-FG02-87ER13808), the NSF (graduate traineeship for R.D.W.), and the Dreyfus Foundation (Teacher-Scholar Award to J.T.H.) for support of this work.

\section{References and Notes}

(1) (a) McManis, G. E.; Nielson, R. M.; Weaver, M. J. J. Am. Chem. Soc. 1989, 111, 5533. (b) Gennett, T.; Miher, D. F.; Weaver, M. J. J. Phys. Chem. 1985, 89, 2787. (c) Nielson, R. M.; Golovin, M. N.; McManis, G. E.; Weaver, M. J. J. Am. Chem. Soc. 1988, 110, 1745. (d) Boukheddaden, K.; Linares, J.; Bousseksou, A.; Nasser, J.; Rabah, H.; Varret, F. Chem. Phys. 1993, 170, 47.

(2) (a) Chidsey, C. E. D. Science 1991, 251, 919. (b) Smalley, J. F.; Chidsey, C. E. D.; Feldberg, S. W.; Newton, M. D.; Liu, Y. P. J. Phys. Chem. 1995, 99, 13141. (c) Weber, K.; Creager, S. E. Anal. Chem. 1994, 66, 3164. (d) Tender, L.; Carter, M. T.; Murray, R. W. Anal. Chem. 1994, 66, 3173.

(3) (a) Morrison, W. H.; Hendrickson, D. N. Inorg. Chem. 1975, 14, 2331. (b) Dong, T. Y.; Hendrickson, D. N.; Iwai, K.; Cohn, M. J.; Geib, S. J.; Rheingold, A. L.; Sano, H.; Motoyama, I.; Nakashima, S. J. Am. Chem. Soc. 1985, 107, 7996. (c) Webb, R. J.; Hagen, P. M.; Wittebort, R. J.; Sorai, M.; Hendrickson, D. N. Inorg. Chem. 1992, 31, 1791. (d) Dong, T.-Y.; Huang, C.-H.; Chang, C.-K.; Hsieh, H.-C.; Peng, S.-M.; Lee, G.-H.; Organometallics 1995, 14, 1776.

(4) (a) Powers, M. J.; Meyer, T. J. J. Am. Chem. Soc. 1978, 100, 4393. (b) McManis, G. E.; Nielson, R. M.; Weaver, M. J. Inorg. Chem. 1988, 27 , 1827. (c) McManis, G. E.; Gochev, A.; Nielson, R. M.; Weaver, M. J. J.
Phys. Chem. 1989, 93, 7733. (d) Nielson, R. M.; McManis, G. E.; Golovin, M. N.; Weaver, M. J. J. Phys. Chem. 1988, 92, 3441.

(5) (a) Blackbourn, R. L.; Hupp, J. T. Chem. Phys. Lett. 1988, 150, 399. (b) Blackbourn, R. L.; Hupp, J. T. J. Phys. Chem. 1990, 94, 1788. (c) Hammack, W. S.; Drickamer, H. G.; Lowery, M. D.; Hendrickson, D. N. Chem. Phys. Lett. 1986, 132, 231. (d) Hammack, W. S.; Drickamer, H. G.; Lowery, M. D.; Hendrickson, D. N. J. Am. Chem. Soc. 1987, 109, 8019.

(6) (a) Nielson, R. M.; Hupp, J. T. Inorg. Chem. 1996, 35, 2032. (b) Nielson, R. M.; Hupp, J. T. Inorg. Chem. 1996, 35, 1402.

(7) Ribou, A.-C.; Launay, J.-P.; Sachtleben, M. L.; Li, H.; Spangler, C. W. Inorg. Chem. 1996, 35, 3735.

(8) Newton, M. D.; Ohta, K.; Zhong, E. J. Phys. Chem. 1991, 95, 2317.

(9) (a) Hush, N. S. Prog. Inorg. Chem. 1967, 8, 391. (b) Creutz, C. Prog. Inorg. Chem. 1983, 30, 1. (c) Sutin, N. Prog. Inorg. Chem. 1983, 30, 441. (d) Sutin, N. Adv. Chem. Ser. 1991, 228, 25. (e) Crutchley, R. J. Adv. Inorg. Chem. 1994, 41, 273.

(10) For reviews, see: (a) Myers, A. B. Chem. Phys. 1994, 180, 215. (b) Shin, K. S. K.; Zink, J. I. Adv. Photochem. 1991, 16, 119. (c) Heller, E. J. Acc. Chem. Res. 1981, 14, 368.

(11) See, for example: Doorn, S. K.; Hupp, J. T. J. Am. Chem. Soc. 1989, 111,1142 .

(12) (a) Petrov, V.; Hupp, J. T.; Mottley, C.; Mann, L. C. J. Am. Chem. Soc. 1994, 116, 2171. (b) Lu, H.; Petrov, V.; Hupp, J. T. Chem. Phys. Lett. 1995, 235, 521. (c) Karki, L.; Williams, R. D.; Hupp, J. T.; Allan, C. B.; Spreer, L. O. Inorg. Chem., submitted.

(13) Doorn, S. K.; Hupp, J. T.; Porterfield, D. R.; Campion, A.; Chase, D. B. J. Am. Chem. Soc. 1990, 112, 4999.

(14) (a) Bodenheimer, J. S.; Low, W. Spectrochim. Acta 1973, 29A, 1733. (b) Kimel'fel'd, Y. M.; Smirnova, E. M. J. Mol. Struct. 1973, 19 329. (c) Lippincott, E. R.; Nelson, R. D. Spectrochim. Acta 1958, 10, 307.

(15) Gächter, B. F.; Konigstein, J. A.; Aleksanjan, V. T. J. Chem. Phys. 1975, 62, 4628.

(16) Interestingly, $v_{21}$ in ferrocene (assuming a $D_{5 d}$ point group) has $\mathrm{E}_{\text {lu }}$ symmetry so that it is IR (but not Raman) allowed, as is found experimentally. However, in a solid-state ferrocenium Raman spectrum, a $510 \mathrm{~cm}^{-1}$ band was assigned to $v_{21}$, presumably because the actual symmetry was lower than $D_{5 d} .^{15}$ Analogously, the less than $D_{5 d}$ symmetry of biferrocene in principle allows $v_{21}$ to become Raman active. This motion may also arise from Jahn-Teller distortion of the ${ }^{2} \mathrm{E}_{2 \mathrm{~g}}$ ground state of ferrocenium (see Discussion).

(17) (a) Berces, A.; Ziegler, T.; Fan, L. J. Phys. Chem. 1994, 98, 1584. (b) Margle, P.; Schwarz, K.; Blöchl, P. E. J. Chem. Phys. 1994, 100, 8194.

(18) A possible assignment of the latter as a stretch of the bond between the ferrocene and ferrocenium halves of the molecule was discounted because the symmetry of the stretch would preclude its being coupled to a charge transfer.

(19) Schomacker, K. T.; Bangcharoenpaurpong, O.; Champion, P. M. J. Chem. Phys. 1984, 80, 4701.

(20) (a) Shreve, A. P.; Mathies, R. A. J. Phys. Chem. 1995, 99, 7285. (b) Page, J. B.; Tonks, D. L. J. Chem. Phys. 1981, 75, 5694.

(21) Marcus, R. A. J. Chem. Phys. 1965, 43, 679.

(22) A slightly smaller value for $\chi_{\mathrm{s}}$ is obtained if the solvent-induced spectral shifts are intrepreted by using a mean spherical approximation model. ${ }^{4 \mathrm{c}}$

(23) Note that the fitting protocol is such that with $\chi_{\mathrm{s}}, E_{00}, T$, and $\Gamma$ independently fixed, the $\Delta_{k}$ 's can be varied until the agreement between calculated and experimental intensities is arbitrarily exact. An exact fitting of experimental Raman intensities, however, is an insufficient criterion to ensure a fully accurate fit.

(24) (a) Hendrickson, D. N.; Sohn, Y. S.; Duggan, D. M.; Gray, H. B. J. Chem. Phys. 1973, 58, 4666. (b) Rowe, M. D.; McCaffrey, A. J. J. Chem. Phys. 1973, 59, 3786.

(25) Stebler, A.; Furrer, A.; Ammeter, A. H. Inorg. Chem. 1984, 23, 3493.

(26) Thermal population of multiple initial states is also conceivable.

(27) For simplicity (and for lack of required information) we neglect any broadening effects from variation in $\chi_{\mathrm{s}}$ or $\chi_{\text {vib }}$ with changes in intervalence excited-state identity. We also neglect any variations in oscillator strength. We further assume (again, for simplicity) that $H_{\mathrm{ab}}$ for the most probable intervalence transition can be estimated ${ }^{26}$ by equating its transition moment with the measured apparent overall transition moment. Obviously, if more than one transition contributes significantly to the observed intervalence absorption intensity, then $H_{\mathrm{ab}}$ will be overestimated.

(28) Cave, R.; Newton, M. D. Chem. Phys. Lett. 1996, 249, 15.

(29) It should be noted that spectral experiments report on adiabatic reorganizational energies. These will be slightly smaller than the corresponding diabatic energies. ${ }^{41}$

(30) Hush, N. S. Coord. Chem. Rev. 1985, 64, 135.

(31) This estimate is based upon an ellipsoidal cavity calculation for $\chi_{\mathrm{s}}$ (Brunschwig, B. S.; et al. J. Phys. Chem. 1986, 90, 3657), rather than the usual two-sphere calculation. ${ }^{21}$ The dimensions of the cavity were motivated by the available crystallographic data ${ }^{36 a}$ (with biferrocene in the trans configuration) and by an "equal volume ellipsoid" approximation. The analysis indicated that a change in dipole moment $(\Delta \mu)$ of 5.1 e $\AA$, corresponding to unit charge transfer over the full metal-metal separation 
distance, would yield a $\chi_{\mathrm{s}}$ value of $4300 \mathrm{~cm}^{-1}$. Diminution of $\Delta u$ to $3.5 \mathrm{e}$ $\AA$, however, reproduced the $\chi_{\mathrm{s}}$ value derived from Pekar plots. ${ }^{4 a}$ We note that Stark spectroscopy studies have recently yielded several examples where effective charge transfer occurs over distances that are substantially less than geometric donor/acceptor separation distances (see, for example: Shin, Y. K.; et al. J. Am. Chem. Soc. 1995, 117, 8668; Oh, D. H.; et al. J. Am. Chem. Soc. 1991, 113, 6880; Karki, L.; Lu, H. P.; Hupp, J. T. J. Phys. Chem. 1996, 100, 15637).

(32) (a) Reber, C.; Zink, J. I. Comments Inorg. Chem. 1992, 13, 177. (b) Reber, C.; Zink, J. I. J. Chem. Phys. 1992, 96, 2681. (c) Reber, C.; Zink, J. I. J. Phys. Chem. 1992, 96, 571. (d) Wooton, J. L.; Zink, J. I. J. Phys. Chem. 1995, 99, 7251.

(33) The presence of multiple excited states with different displacements in each may also lead to interference effects (see, for example: Pfennig, B. W.; Wu, Y.; Kumble, R.; Spiro, T. G.; Bocarsly, A. B. J. Phys. Chem. 1996, 100,5745 , and ref 18 a therein.).

(34) (a) Newton, M. D.; Sutin, N. Annu. Rev. Phys. Chem. 1984, 35, 437. (b) Landau, L. Phys. Z. Sowjetunion 1932, 2, 46. (c) Zener, C. Proc. R. Soc. London, Ser. A 1932, 137, 696; 1933, 140, 660.

(35) The transition probability was taken to be ${ }^{9 \mathrm{c}, 34 a} P_{12}=1-[1-$ $\left.\exp \left(-v_{\mathrm{el}} / 2 v_{n}\right)\right]$, where $v_{\mathrm{el}}=\left(2 H_{\mathrm{ab}}{ }^{2} / h\right)\left(\pi^{3} / \chi R T\right)^{1 / 2}$. A temperature of $298 \mathrm{~K}$ was used. For simplicity, we implemented the calculation as a single-mode problem; that is, the entire reorganization energy $\left(\chi=4950 \mathrm{~cm}^{-1}\right)$ was ascribed to the $1100 \mathrm{~cm}^{-1}$ mode $\left(v_{n}\right)$. While this obviously is inaccurate, it should provide a useful upper limit estimate for the crossing probability.

(36) (a) Geib, S. J.; Rheingold, A. L.; Dong, T.; Hendrickson, D. N. J. Organomet. Chem. 1986, 312, 241. (b) Webb, R. J.; Rheingold, A. L.; Geib, S. J.; Staley, D. L.; Hendrickson, D. N. Angew. Chem., Int. Ed. Engl. 1989, 28, 1388. (c) Konno, M.; Hyodo, S.; Iijima, S. Bull. Chem. Soc. Jpn. 1982, $55,2327$.

(37) Conversion from differences in crystallographic bond length $(\Delta a)$ to normal coordinate displacements requires a knowledge of the vibrational frequency (obtained from the Raman measurements), the number of equivalent bonds displaced, and the reduced mass..$^{1, c}$ Rearranging eq 8 and equations given by Weaver (and combining constants), we obtain the following: $\Delta=0.1723(\Delta a)(b v \mu)^{1 / 2}$, where $\Delta$ is the dimensionless displacement, $v$ is the vibrational frequency in wavenumbers, $\mu$ is the reduced mass in amu (taken to be the mass of one cp ring), and $b$ is the number of equivalent bonds displaced (taken to be 4).

(38) (a) Brunschwig, B. S.; Sutin, N. Comments Inorg. Chem. 1987, 6, 209. (b) Spears, K. G. J. Phys. Chem. 1995, 99, 2469. (c) Jortner, J.; Bixon, M. J. J. Chem. Phys. 1987, 88, 167. (d) Sarai, A.; Kakitani, T. Chem. Phys. Lett. 1981, 77, 427.

(39) In the equation, $S$ equals $\Delta^{2} / 2, m$ is the change in vibrational quantum number, $I_{m}$ is a modified Bessel function of order $m$, and $\Delta E$ is the difference in energy between the initial state and the final state. The first and third summations and the product term encompass all modes that are Franck-Condon active, while the quantum number summation (second summation), though technically infinite, generally converges if levels between -10 and +10 are considered.

(40) If the assignment of the $504 \mathrm{~cm}^{-1}$ mode as an IR active stretch of ferrocene is correct, this mode should be omitted from the comparison of outer-sphere reaction rates (unless, of course, such reactions occur from a precursor state that entails similar symmetry reduction).

(41) In the diabatic limit, $\chi$ (total) will increase by an amount sufficient to compensate exactly for the loss of contributions from $E$ (split) to the vertical separation energy. In other words, $E_{\mathrm{op}}=\chi($ total,diabatic $)+E_{00}=$ $\chi$ (total,nonadiabatic) $+E$ (split) $+E_{00}$. If $\chi$ (total,diabatic) is employed in eq 10 , then $k_{\text {solvent }} / k$ (solvent +3 Raman modes) increases from 31.9 to 32.3 under classical conditions (cf. Table 2). For further discussion, see: Creutz, C.; Newton, M. D.; Sutin, N. J. Photochem. Photobiol. A 1994, 82, 47.

(42) In retrospect, bond distortions corresponding to the additional normal mode displacements likely could have been extracted from crystallographic data. Connecting these many-atom distortions to mode-specific reorganization energy contributions, however, would generally be difficult. These difficulties, and a well-founded traditional emphasis on metal-ligand stretching effects in transition metal based redox systems, no doubt account for neglect of other modes in prior ET-related evaluations of crystallographic data. 